Zou, H.L., and Abdel-Malek, K., and Wang, J.Y., (1997), "A Variational Approach for the Design of the Spatial Four-Bar Mechanism". Mechanics of Structures of Machines, Vol. 25, No. 1, pp. 41-59.

\title{
A Variational Approach for the Design of the Spatial Four-Bar Mechanism
}

\author{
H. L. Zou \\ K. A. Abdel-Malek \\ J. Y. Wang
}

Center for Computer Aided Design and Department of Mechanical Engineering

The University of Iowa

Iowa City, IA 52242

\section{ABSTRACT}

An analytical formulation for computing kinematic sensitivity of the spatial four-bar mechanism is presented. The experimental code developed is used to compute an assembled configuration for the mechanism due to a design variation. The mechanism is modeled using graph theory where a body is defined as a node and a kinematic joint is defined as an edge. The spherical joint is cut to convert the model into a tree structure by cutting an edge and introducing constraints. The effect of variation in mechanism design using concepts of virtual displacement and rotation is introduced. The variation of the spherical constraint is computed while maintaining joint-attachment vectors and orientation matrices as variables. A system of equations that has more design variables than equations is then solved using the modified Moore-Penrose pseudo inverse. A recursive formulation is introduced to obtain the state variation of a body in terms of the state variation of a junction body and of the relative coordinates along the chain. The Jacobian matrix is then transformed from Cartesian coordinate space to joint coordinate space using velocity transformation matrices. Kinematic sensitivity analyses due to changing a jointattachment vector and an orientation are presented.

\section{INTRODUCTION}

The planar and spatial four-bar mechanisms are some of the most used machine subsystems. Because the concept of these mechanisms is old and widely used, they have been extensively studied. In recent years, the emphasis has been on the synthesis using numerical techniques. For example, the finite position synthesis of a spherical four-bar mechanism using a mapping approach of the desired positions to points in an image space was presented by Bodduluri and McCarthy [1]. A solution of the nine-point path synthesis of four-bar linkages was presented by Wampler, et al. [2] where the problem is formulated and solved using a combination of classical elimination, multihomogeneous variables, and numerical continuation methods. Another method that uses the continuation method in the analysis of four-bar linkages was reported by Subbian and Flugrad [3]. Graphical synthesis of four-bar linkages were reported by Norton, et al. [4] and Khanuja, et al. [5]. Other synthesis methods are reported by Hwang and Chang [6]. 
Computer-aided design techniques were also used to automate the infinite point coupler curve synthesis using uniform periodic B-splines [7]. The geometry and dynamics of the configuration space of the four-bar mechanism using singularity theory was studied by Yang and Krishnaprasad [8].

Works that have dealt with the transmission angle of the four-bar mechanism in terms of the mechanism's mobility analysis were presented by Kazerounian and Solecki [9]. The mechanism's constant force transmission characteristics were studied by Soylemez [10]. The input-output equation of the underlying mechanism was analytically studied for constant-branch motion by Liu and Angeles [11] who also studied the least square optimization of planar and spherical mechanisms under mobility constraints [12]. A similar analysis was applied to the mechanism's path generators [13].

Sheth and Uicker [14] implemented graph theory to analyze the topology of multibody systems in terms of relative coordinates. Graph theory was also used by Wittenburg [15] to handle closed loop systems by cutting joints to form a spanning tree. Wittenburg and Wolz [16] also presented a cut-body method in a computer program for articulated multibody dynamics. The recursive formulation used in this paper was adapted from the work of Bae and Haug [17, 18] who used Wittenburg's approach to modeling of multibody systems. This type of formulation was implemented using parallel computational techniques by Hwang, et al. [19] and Hwang and Haug [20].

\section{CONCEPT OF VARIATION IN MECHANISM COMPONENT DESIGN USING VIRTUAL DISPLACEMENTS AND ROTATIONS}

A spatial four-bar mechanism shown in Fig. 1 is modeled as a four-body system connected by a spherical, a universal, and two revolute joints. The ground is chosen as the base body where its body-fixed reference frame coincides with the global reference frame.

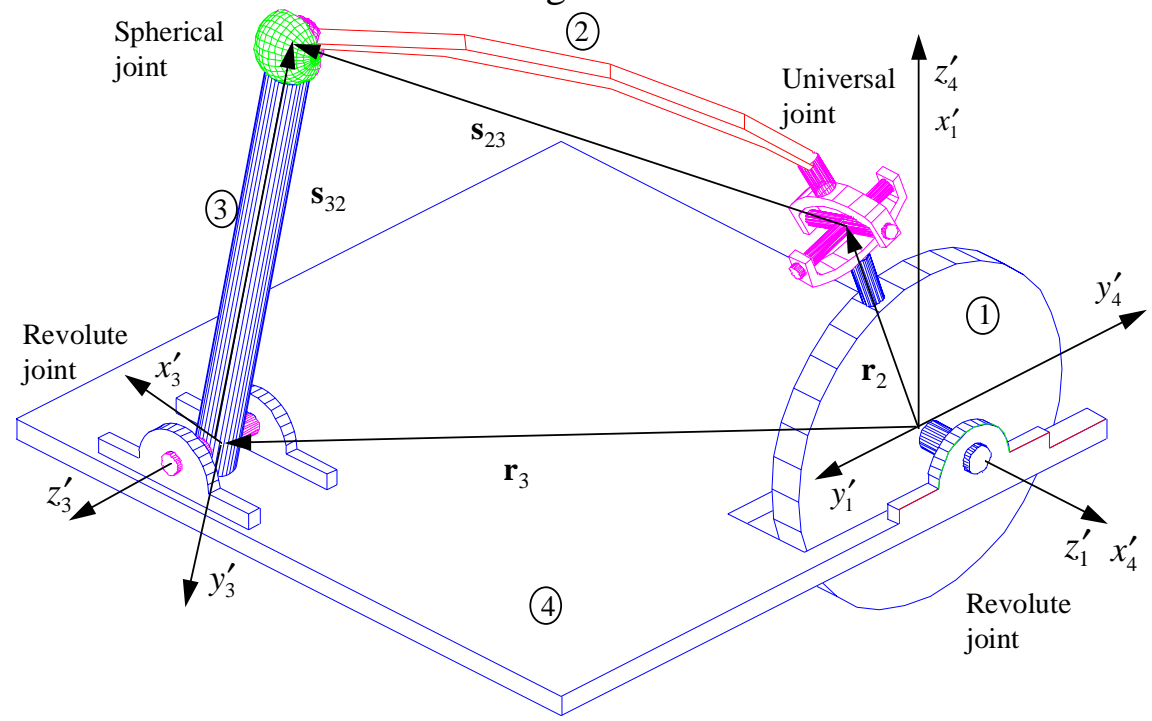

Fig. 1 Spatial four-bar mechanism 
The spanning tree of the system is shown in Fig. 2 where the spherical joint has been cut. Cutting the spherical joint reduces the maximum number of relative coordinates (three) and introduces a minimum number of cut-joint constraints. Two relative coordinates $q_{1}$ (between the ground and link 1) and $q_{4}$ (between link 3 and the ground) are used for the two revolute joints. Two coordinates $q_{2}$ and $q_{3}$ represent the universal joint connecting link 1 and link 2 . A bodyfixed frame $x_{i}^{\prime} y_{i}^{\prime} z_{i}^{\prime}$ is defined at the joint connected to the inboard body of the kinematic chain, and the coordinate systems $x_{i}^{\prime \prime} y_{i}^{\prime \prime} z_{i}^{\prime \prime}$ are defined at joints that are connected to the outboard bodies.

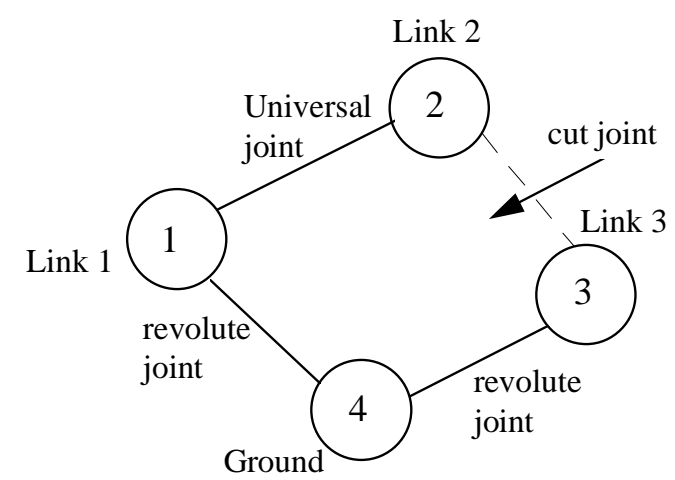

Fig. 2 (a) Graphic representation of the spatial four-bar mechanism

In order to develop the formulation for the cut spherical joint, it is convenient to write the spherical constraint as a requirement that necessitates a pair of points on two bodies to coincide. A necessary and sufficient condition for $O_{i j}^{\prime \prime}$ and $O_{j i}^{\prime \prime}$ to coincide [21] is that $\mathbf{d}_{i j}=\mathbf{0}$ such that

$$
\Phi^{s p h}\left(O_{i j}^{\prime \prime}, O_{j i}^{\prime \prime}\right)=\mathbf{r}_{j}+\mathbf{s}_{j i}-\mathbf{r}_{i}-\mathbf{s}_{i j}=\mathbf{0}
$$

where $\Phi^{s p h}$ denotes a vector of constraint functions for the spherical joint. The three scalar constraint equations of the spherical joint are defined as

$$
\Phi^{s p h}=\mathbf{r}_{3}+\mathbf{s}_{32}-\mathbf{r}_{2}-\mathbf{s}_{23}=\mathbf{0}
$$

Since the spatial four-bar mechanism has four generalized coordinates and three constraint equations, it has one degree-of-freedom. In deriving the variational constraints, it is necessary to keep the joint-attachment vectors and the orientation matrices as variables in order to later obtain their kinematic sensitivity.

\section{VARIATION IN MECHANISM COMPONENT DESIGN USING VIRTUAL DISPLACEMENT AND ROTATION}

In this section, the concept of virtual displacement and rotation will be introduced. In order to account for the effect of design variation, it is necessary to develop a formulation for the constraint variation while maintaining variable parameters. The virtual displacement and rotation vectors (joint-attachment vectors and orientation matrices) will be maintained as variables in order to later obtain their sensitivity. The study of the effect of design change on kinematic variables and the study of design change required to preserve kinematic configurations will be addressed and demonstrated via numerical examples. 
Since the spherical constraint of the four-bar mechanism is cut, consider its variation as

$$
\delta \Phi^{s p h}=\delta \mathbf{r}_{j}+\delta \mathbf{s}_{j i}-\delta \mathbf{r}_{i}-\delta \mathbf{s}_{i j}
$$

where joint-attachment vectors can be resolved in the body reference frame as

$$
\mathbf{s}_{i j}=\mathbf{A}_{i} \mathbf{s}_{i j}^{\prime}
$$

where $\mathbf{A}_{i}$ is a transformation matrix from the body coordinate system $x_{i}^{\prime} y_{i}^{\prime} z_{i}^{\prime}$ to the global coordinate system $x y z$. Taking the variation of both sides of Eq. (4) yields

$$
\delta \mathbf{s}_{i j}=\delta \mathbf{A}_{i} \mathbf{s}_{i j}^{\prime}+\mathbf{A}_{i} \delta \mathbf{s}_{i j}^{\prime}
$$

Note that $\delta \mathbf{s}_{i j}^{\prime}$ and $\delta \delta_{j i}^{\prime}$ are variables. The virtual rotation matrix $\delta \tilde{\pi}$ (a skew-symmetric matrix) was defined by Tsai and Haug [22] as

$$
\delta \tilde{\pi}=\delta \mathbf{A A}^{T}
$$

where the tilde operator (i.e., the symbol $\sim$ ) is used to denote a skew-symmetric matrix generated by the associated vector. Similar to the virtual rotation matrix $\delta \tilde{\pi}$ associated with the matrix $\mathbf{A}$, define a virtual rotation matrix $\delta \tilde{\xi}^{\prime}$ associated with the transformation matrix $\mathbf{C}_{i j}$ such that

$$
\delta \tilde{\xi}_{i j}^{\prime}=\delta \mathbf{C}_{i j} \mathbf{C}_{i j}^{T}
$$

where $\mathbf{C}_{i j}$ is the transformation matrix from joint reference frame $x^{\prime \prime} y^{\prime \prime} z^{\prime \prime}$ to body reference frame $x^{\prime} y^{\prime} z^{\prime}$. To simplify the computation of virtual rotations, Haug [21] suggested using the the virtual Euler parameters to represent $\delta \xi_{i j}$ such that

$$
\delta \xi_{i j}=2 \mathbf{A}_{i} \mathbf{E}_{j} \delta \mathbf{p}_{j}
$$

where $\mathbf{p}_{j}=\left[\begin{array}{ll}e_{0} & \mathbf{e}\end{array}\right]^{T}=\left[\begin{array}{llll}e_{0} & e_{1} & e_{2} & e_{3}\end{array}\right]^{T}$ is the vector of Euler parameters $\left(e_{j}\right)$ and $\mathbf{E}_{j}$ is the Euler semi-rotation matrix defined as

$$
\mathbf{E}_{j}=\left[\begin{array}{cccc}
-e_{1} & e_{0} & -e_{3} & e_{2} \\
-e_{2} & e_{3} & e_{0} & -e_{1} \\
-e_{3} & -e_{2} & e_{1} & e_{0}
\end{array}\right]
$$

For example, $\mathbf{p}_{3}$ is the vector of Euler parameters associated with $\mathbf{C}_{43}$.

Multiplying Eq. (6) from the left by $\mathbf{A}$ yields another property

$$
\delta \tilde{\pi} \mathbf{A}=\delta \mathbf{A}
$$

Using the relationships of virtual displacement and rotation, Eq. (5) can be written in terms of the virtual vectors as

$$
\delta \mathbf{s}_{i j}=-\widetilde{\mathbf{s}}_{i j} \delta \pi_{i}+\mathbf{A}_{i} \delta \mathbf{s}_{i j}^{\prime}
$$

Substituting the expressions for $\delta_{i j}$ into Eq. (3) yields the variation of the spherical constraint as

$$
\delta \Phi^{s p h}=\delta \mathbf{r}_{j}-\delta \mathbf{r}_{i}-\widetilde{\mathbf{s}}_{j i} \delta \pi_{j}+\mathbf{A}_{j} \delta \mathbf{s}_{j i}^{\prime}+\widetilde{\mathbf{s}}_{i j} \delta \pi_{i}-\mathbf{A}_{i} \delta \mathbf{s}_{i j}^{\prime}
$$

The constraint equation written in general form is

$$
\Phi\left(\mathbf{r}_{i}, \mathbf{A}_{i}, \mathbf{s}_{i j}, \mathbf{C}_{i j}, \mathbf{r}_{j}, \mathbf{A}_{j}, \mathbf{C}_{j i}, \mathbf{s}_{j i}\right)=\mathbf{0}
$$

Noting that $\mathbf{s}$ and $\mathbf{C}$ are variables, the variation of Eq. (13) yields

$$
\delta \Phi=\Phi_{\mathbf{r}_{i}} \delta \mathbf{r}_{i}+\Phi_{\pi_{i}} \delta \pi_{i}+\Phi_{\mathbf{r}_{j}} \delta \mathbf{r}_{j}+\Phi_{\pi_{j}} \delta \pi_{j}+\Phi_{\mathbf{s}_{i j}^{\prime}} \delta \mathbf{s}_{i j}^{\prime}+\Phi_{s_{j i}^{\prime}} \delta \mathbf{s}_{j i}^{\prime}
$$




$$
+\Phi_{\xi_{i j}} \delta \xi_{i j}+\Phi_{\xi_{j i}} \delta \xi_{j i}=\mathbf{0}
$$

Furthermore, the linearized constraint of Eq. (14) can be written as

$$
\delta \Phi=\Phi_{\mathbf{z}_{i}} \delta \mathbf{z}_{i}+\Phi_{\mathbf{z}_{j}} \delta \mathbf{z}_{j}+\Phi_{\xi^{i j}} \delta \xi^{i j}+\Phi_{\mathbf{s}^{j}} \delta^{i j}=\mathbf{0}
$$

where the extended vector of the virtual joint-attachment vector is

$$
\delta \mathbf{s}^{i j}=\left[\begin{array}{l}
\delta \mathbf{s}_{i j}^{\prime} \\
\delta \mathbf{s}_{j i}^{\prime}
\end{array}\right]
$$

and the virtual rotation is

$$
\delta \xi^{i j}=\left[\begin{array}{l}
\delta \xi_{i j} \\
\delta \xi_{j i}
\end{array}\right]
$$

the coefficients of virtual displacement are

$$
\begin{aligned}
\Phi_{\mathbf{z}_{i}} & =\left[\begin{array}{ll}
\Phi_{\mathbf{r}_{i}} & \Phi_{\pi_{i}}
\end{array}\right] \\
\Phi_{\mathbf{z}_{j}} & =\left[\begin{array}{ll}
\Phi_{\mathbf{r}_{j}} & \Phi_{\pi_{j}}
\end{array}\right]
\end{aligned}
$$

the coefficients of virtual joint-attachment and rotation are

$$
\begin{aligned}
\Phi_{\mathbf{s}^{i j}} & =\left[\begin{array}{ll}
\Phi_{\mathbf{s}_{i j}^{\prime}} & \Phi_{\mathbf{s}_{j_{i j}}}
\end{array}\right] \\
\Phi_{\xi^{i j}} & =\left[\begin{array}{ll}
\Phi_{\xi_{\xi_{j}}} & \Phi_{\xi_{j_{i j}}}
\end{array}\right]
\end{aligned}
$$

and the virtual displacement for bodies $i$ and $j$ are

$$
\delta \boldsymbol{z}_{\mathrm{i}}=\left[\begin{array}{c}
\delta \mathbf{r}_{\mathrm{i}} \\
\delta \pi_{\mathrm{i}}
\end{array}\right] \quad \delta \mathbf{z}_{\mathrm{j}}=\left[\begin{array}{l}
\delta \mathbf{r}_{\mathrm{j}} \\
\delta \pi_{\mathrm{j}}
\end{array}\right]
$$

Note that the variation of the constraint in Eq. (15) is an extended expression of the formulation used by Tsai and Haug [22], where the last two terms of the right-hand side are used to account for the variation in joint-attachment and orientation vectors.

\section{RECURSIVE FORMULATION}

In order to develop a formulation for obtaining the variation of the constraint function $\Phi$ in terms of the variation of the generalized joint coordinates including the effect of design variation, Tsai and Haug [22] proposed writing the constraint variation in the form of

$$
\delta \Phi=\Phi_{\mathbf{q}} \delta \mathbf{q}
$$

where $\delta \mathbf{q}$ is the vector of relative coordinate variations and $\Phi_{\mathbf{q}}$ is the Jacobian matrix in Joint space. In this work, the system of equations (Eq. 23) includes the virtual displacement and rotation vectors as variables. A formulation to recursively obtain the state variation of body $i$ in terms of the state variation of its junction body and of the relative coordinates along the chain will be developed. The system of equations (Eq. 23) may have more unknowns than constraints. The Moore-Penrose pseudo inverse is then used to obtain an assembled configuration close to the initial guess provided by a user.

The orthogonal transformation matrix $\mathbf{A}_{j}$ from the body $j$ reference frame to the global reference frame can be written as 


$$
\mathbf{A}_{j}=\mathbf{A}_{i} \mathbf{C}_{i j} \mathbf{A}_{i j}^{\prime \prime}
$$

where $\mathbf{A}_{i j}^{\prime \prime}$ is the transformation matrix from the body $j$ reference frame $x_{j}^{\prime} y_{j}^{\prime} z_{j}^{\prime}$ to the joint reference frame of body $i\left(x_{i j}^{\prime \prime} y_{i j}^{\prime \prime} z_{i j}^{\prime \prime}\right)$. Taking the variation of Eq. (24) yields

$$
\delta \mathbf{A}_{j}=\delta \mathbf{A}_{i} \mathbf{C}_{i j} \mathbf{A}_{i j}^{\prime \prime}+\mathbf{A}_{i} \delta \mathbf{C}_{i j} \mathbf{A}_{i j}^{\prime \prime}+\mathbf{A}_{i} \mathbf{C}_{i j} \delta \mathbf{A}_{i j}^{\prime \prime}
$$

Substituting Eqs. (6) and (10) into Eq. (25) and multiplying by the virtual rotation matrix $\delta \tilde{\pi}_{j}$ yields

$$
\delta \tilde{\pi}_{j} \mathbf{A}_{j}=\delta \tilde{\pi}_{i} \mathbf{A}_{i} \mathbf{C}_{i j} \mathbf{A}_{i j}^{\prime \prime}+\mathbf{A}_{i} \delta \tilde{\xi}_{i j}^{\prime} \mathbf{C}_{i j} \mathbf{A}_{i j}^{\prime \prime}+\mathbf{A}_{i} \mathbf{C}_{i j} \delta \tilde{\pi}_{i j}^{\prime} \mathbf{A}_{i j}^{\prime \prime}
$$

where $\delta \pi_{i j}$ can be obtained from the relative coordinate variation $\delta \mathbf{q}_{j}$, such that

$$
\delta \pi_{i j}=\mathbf{H}_{j}\left(\mathbf{A}_{i}, \mathbf{q}_{j}\right) \delta \mathbf{q}_{j}
$$

and $\mathbf{H}_{j}\left(\mathbf{A}_{i}, \mathbf{q}_{j}\right)$ is a transformation matrix that depends on the orientation of body $i$ and the relative coordinates $\mathbf{q}_{j}$, which is defined for each type of joint. It can also be shown that $\delta \pi_{i j}$ can be written as

$$
\delta \pi_{j}=\delta \pi_{i}+\delta \xi_{i j}+\mathbf{H}_{j} \delta \mathbf{q}_{j}
$$

Referring to Fig. 1, the origin of body $j$ reference frame can be located by the position vector given by

$$
\mathbf{r}_{j}=\mathbf{r}_{i}+\mathbf{s}_{i j}+\mathbf{d}_{i j}
$$

The variation of Eq. (29) was derived by Zou, et al. [23] and shown to be

$$
\delta \mathbf{r}_{j}=\delta \mathbf{r}_{i}+\delta \widetilde{\pi}_{i}\left(\mathbf{r}_{j}-\mathbf{r}_{i}\right)+\mathbf{A}_{i} \delta \mathbf{s}_{i j}^{\prime}-\widetilde{\mathbf{d}}_{i j} \delta \xi_{i j}+\frac{\partial \mathbf{d}_{i j}}{\partial \mathbf{q}_{j}} \delta \mathbf{q}_{j}
$$

Adding a common term $\left(\tilde{\mathbf{r}}_{j} \delta \pi_{j}\right)$ to both sides of Eq. (30), and using Eq. (6), Eq. (28), and the relationship $\tilde{\mathbf{a}} \mathbf{b}=-\tilde{\mathbf{b}} \mathbf{a}$ yields

$$
\delta \mathbf{r}_{j}+\widetilde{\mathbf{r}}_{j} \delta \pi_{j}=\delta \mathbf{r}_{i}+\widetilde{\mathbf{r}}_{i} \delta \pi_{i}+\mathbf{A}_{i} \delta \mathbf{s}_{i j}^{\prime}-\widetilde{\mathbf{d}}_{i j} \delta \xi_{i j}+\left(\frac{\partial \mathbf{d}_{i j}}{\partial \mathbf{q}_{j}}+\widetilde{\mathbf{r}}_{j} \tilde{\pi}_{j}\right) \delta \mathbf{q}_{j}+\widetilde{\mathbf{r}}_{j} \delta \xi_{i j}
$$

Equation (31) and the virtual rotation of Eq. (28) can be combined in matrix form as

$$
\left[\begin{array}{c}
\delta \mathbf{r}_{j}+\widetilde{\mathbf{r}}_{j} \delta \pi_{j} \\
\delta \pi_{j}
\end{array}\right]=\left[\begin{array}{c}
\delta \mathbf{r}_{i}+\widetilde{\mathbf{r}}_{i} \delta \pi_{i} \\
\delta \pi_{i}
\end{array}\right]+\left[\begin{array}{c}
\mathbf{A}_{i} \\
\mathbf{0}
\end{array}\right] \delta \mathbf{s}_{i j}^{\prime}+\left[\begin{array}{c}
\frac{\partial \mathbf{d}_{i j}}{\partial \mathbf{q}_{j}}+\widetilde{\mathbf{r}}_{j} \mathbf{H}_{j} \\
\mathbf{H}_{j}
\end{array}\right] \delta \mathbf{q}_{j}+\left[\begin{array}{c}
\widetilde{\mathbf{r}}_{j}-\widetilde{\mathbf{d}}_{i j} \\
\mathbf{I}
\end{array}\right] \delta \xi_{i j}
$$

The tree graph of Fig. (2) represents a pair of links that are disconnected after cutting a joint. First, we shall develop the general formulation for such a case where a joint that connects bodies $i$ and $j$ is cut and that body $p$ is the junction node of the two chains that contain bodies $i$ and $j$, respectively. A schematic is depicted in Fig. 3. 


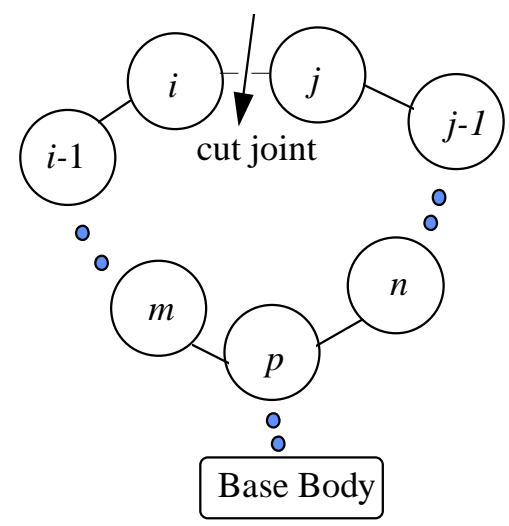

Fig. 3. A pair of bodies is disconnected after cutting joints

In this case, the state variation of body $j$ cannot be represented in terms of the state variation of body $i$ since no relative coordinates exist between bodies $i$ and $j$. Therefore, the state variations of bodies $i$ and $j$ must be written in terms of the state variation of their common node $p$ and Eq. (15) can be written as

$$
\delta \Phi=\Phi_{\hat{\mathbf{z}}_{i}} \delta \hat{\mathbf{z}}_{i}+\Phi_{\hat{\mathbf{z}}_{j}} \delta \hat{\mathbf{z}}_{j}+\Phi_{\xi^{i j}} \delta \xi^{i j}+\Phi_{\mathbf{s}^{i j}} \delta \mathbf{s}^{i j}=\mathbf{0}
$$

where the state vector can be written as

$$
\delta \tilde{\mathbf{z}}_{i}=\delta \tilde{\mathbf{z}}_{i-1}+\mathbf{B}_{i} \delta \mathbf{q}_{i}+\mathbf{M}_{i-1} \delta \mathbf{s}_{i-1, i}^{\prime}+\mathbf{N}_{i} \delta \xi_{i-1, i}
$$

where $\mathbf{B}_{i}$ is the velocity transformation matrix between bodies $i$ and $j$, defined by

$$
\mathbf{B}_{i}=\left[\frac{\partial \mathbf{d}_{(i-1), i}}{\partial \mathbf{q}_{i}}+\tilde{\mathbf{r}}_{i} \mathbf{H}_{i}\right]
$$

and the matrices $\mathbf{M}_{i-1}$ and $\mathbf{N}_{i}$ are expressed as

and

$$
\begin{gathered}
\mathbf{M}_{i-1}=\left[\begin{array}{c}
\mathbf{A}_{i-1} \\
\mathbf{0}
\end{array}\right] \\
\mathbf{N}_{i}=\left[\begin{array}{c}
\widetilde{\mathbf{r}}_{i}-\widetilde{\mathbf{d}}_{(i-1), i} \\
\mathbf{I}
\end{array}\right]
\end{gathered}
$$

Equation (34) can be recursively used to obtain the state variation of body $i$ in terms of the state variation of its common body $p$ and of the relative coordinates along the chain as

$$
\delta \tilde{\mathbf{z}}_{i}=\delta \tilde{\mathbf{z}}_{i-2}+\mathbf{B}_{i-1} \delta \mathbf{q}_{i-1}+\mathbf{M}_{i-2} \delta \mathbf{s}_{i-2, i-1}^{\prime}+\mathbf{N}_{i-1} \delta \xi_{i-2, i-1}+\mathbf{B}_{i} \delta \mathbf{q}_{i}+\mathbf{M}_{i-1} \delta \mathbf{s}_{i-2, i-1}^{\prime}+\mathbf{N}_{i} \delta \xi_{i-1, i}
$$

Simplifying and writing in terms of a series yields

$$
\delta \tilde{\mathbf{z}}_{i}=\delta \hat{\mathbf{z}}_{p}+\sum_{k=m}^{i}\left(\mathbf{B}_{k} \delta \mathbf{q}_{k}+\mathbf{M}_{k-1} \delta \mathbf{s}_{k-1, k}^{\prime}+\mathbf{N}_{k} \delta \xi_{k-1, k}\right)
$$

Similarly, the state variation of body $j$ can be written in terms of a series as 


$$
\delta \hat{\mathbf{z}}_{j}=\delta \hat{\mathbf{z}}_{p}+\sum_{k=n}^{j}\left(\mathbf{B}_{k} \delta \mathbf{q}_{k}+\mathbf{M}_{k-1} \delta \mathbf{s}_{k-1, k}^{\prime}+\mathbf{N}_{k} \delta \boldsymbol{\xi}_{k-1, k}\right)
$$

Rewriting the constraint function in terms of state vectors yields

$$
\begin{aligned}
\delta \Phi & =\Phi_{\hat{\mathbf{z}}_{i}}\left[\delta \hat{\mathbf{z}}_{p}+\sum_{k=m}^{i}\left(\mathbf{B}_{k} \delta \mathbf{q}_{k}+\mathbf{M}_{k-1} \delta \mathbf{s}_{k-1, k}^{\prime}+\mathbf{N}_{k} \delta \xi_{k-1, k}\right)\right] \\
& +\Phi_{\hat{\mathbf{z}}_{j}}\left[\delta \hat{\mathbf{z}}_{p}+\sum_{k=n}^{j}\left(\mathbf{B}_{k} \delta \mathbf{q}_{k}+\mathbf{M}_{k-1} \delta \mathbf{s}_{k-1, k}^{\prime}+\mathbf{N}_{k} \delta \xi_{k-1, k}\right)\right]+\Phi_{\mathbf{s}^{i j}} \delta \mathbf{s}^{i j}+\Phi_{\xi^{i j}} \delta \xi^{i j}
\end{aligned}
$$

Collecting similar terms and rearranging yields

$$
\begin{aligned}
\delta \Phi= & \Phi_{\hat{\mathbf{z}}_{i}} \sum_{k=m}^{i}\left(\mathbf{B}_{k} \delta \mathbf{q}_{k}+\mathbf{M}_{k-1} \delta \mathbf{s}_{k-1, k}^{\prime}+\mathbf{N}_{k} \delta \xi_{k-1, k}\right) \\
& +\Phi_{\hat{\mathbf{z}}_{j}} \sum_{k=n}^{j}\left(\mathbf{B}_{k} \delta \mathbf{q}_{k}+\mathbf{M}_{k-1} \delta \mathbf{s}_{k-1, k}^{\prime}+\mathbf{N}_{k} \delta \xi_{k-1, k}\right)+\Phi_{\mathbf{s}^{i j}} \delta \mathbf{s}^{i j}+\Phi_{\xi^{i j}} \delta \xi^{i j}
\end{aligned}
$$

where $\Phi_{\hat{\mathbf{z}}_{i}}+\Phi_{\hat{\mathbf{z}}_{j}}=\mathbf{0}$ was used to eliminate the $\delta \hat{\mathbf{z}}_{p}$ term in Eq. (41). Expanding Eq. (42) and writing the system of equations in the form of a Jacobian $\Phi_{q}$ yields

$$
\delta \Phi=\Phi_{\mathbf{q}} \delta \mathbf{q}
$$

where the Jacobian matrix in the joint space and the vector of relative coordinate variation $\delta \mathbf{q}$ are defined by

$$
\begin{array}{r}
\Phi_{\mathbf{q}}=\left[\Phi_{\hat{\mathbf{z}}_{i}} \mathbf{B}_{m} \ldots \Phi_{\hat{\mathbf{z}}_{i}} \mathbf{B}_{i}\left|\Phi_{\hat{\mathbf{z}}_{i}} \mathbf{M}_{m-1} \ldots \Phi_{\hat{\mathbf{z}}_{i}} \mathbf{M}_{i-1}\right| \Phi_{\hat{\mathbf{z}}_{i}} \mathbf{N}_{m} \ldots \Phi_{\hat{\mathbf{z}}_{i}} \mathbf{N}_{i} \mid \Phi_{\hat{\mathbf{z}}_{j}} \mathbf{B}_{m} \ldots\right. \\
\Phi_{\hat{\mathbf{z}}_{j}} \mathbf{B}\left|\Phi_{\hat{\mathbf{z}}_{j}} \mathbf{M}_{n-1} \ldots \Phi_{\hat{\mathbf{z}}_{j}} \mathbf{M}_{j-1}\right| \Phi_{\hat{\mathbf{z}}_{j}} \mathbf{N}_{n} \ldots \Phi_{\hat{\mathbf{z}}_{j}} \mathbf{N}_{j}\left|\Phi_{\mathbf{s}^{j j}}\right| \Phi_{\xi^{i j}} \rrbracket
\end{array}
$$

and

$$
\begin{array}{r}
\delta \mathbf{q}=\left[\delta \mathbf{q}_{m} \ldots \delta \mathbf{q}_{i}\left|\delta \mathbf{s}_{m-1, m}^{\prime} \ldots \delta \mathbf{s}_{i-1, i}^{\prime}\right| \delta \xi_{m-1, m} \ldots \delta \xi_{i-1, i}\left|\delta \mathbf{q}_{n} \ldots \delta \mathbf{q}_{j}\right|\right. \\
\left.\delta \mathbf{s}_{n-1, n}^{\prime} \ldots \delta \mathbf{s}_{j-1, j}^{\prime}\left|\delta \xi_{n-1, n}^{\prime} \ldots \delta \xi_{j-1, j}^{\prime}\right| \delta \mathbf{s}^{i j} \mid \delta \xi^{i j}\right]^{T}
\end{array}
$$

Note that the number of variables will, in general, be more than the number of constraints. Since the Jacobian of $\Phi(\mathbf{q})$ is not square, the problem of obtaining an assembled configuration can be solved using the Moore-Penrose pseudo inverse (Allgower and Georg 1990). Starting with an initial guess $\mathbf{q}^{1}$, the new generalized coordinates are calculated by evaluating

$$
\Delta \mathbf{q}=\Phi_{\mathbf{q}}^{*}(-\Phi)
$$

where $\Phi_{\mathbf{q}}^{*}$ is the Moore-Penrose pseudo inverse of the Jacobian $\Phi_{\mathbf{q}}=\left[\partial \Phi_{i} / \partial q_{j}\right]$, defined by

$$
\Phi_{\mathbf{q}}^{*}=\Phi_{\mathbf{q}}^{T}\left(\Phi_{\mathbf{q}} \Phi_{\mathbf{q}}^{T}\right)^{-1}
$$

The new set of generalized coordinates is computed as $\mathbf{q}^{i+1}=\mathbf{q}^{i}+\Delta \mathbf{q}^{i}$. This method converges to an assembled configuration $\mathbf{q}^{*}$ within a few iterations (Allgower and Georg 1990).

\section{EVALUATION OF KINEMATIC SENSITIVITY TERMS}


Initial position and orientation estimates are specified. The initial value of each joint attachment vector $\mathbf{s}_{i j}^{\prime}$ from joint reference frame $x_{i j}^{\prime \prime} y_{i j}^{\prime \prime} z_{i j}^{\prime \prime}$ to body-fixed frame $x_{i}^{\prime} y_{i}^{\prime} z_{i}^{\prime}$ are presented in Table 1.

TABLE 1

Joint attachment vectors $\mathbf{s}_{i j}^{\prime}$ in the body-fixed frame

\begin{tabular}{|lccc|lccc|}
\hline $\mathbf{s}_{i j}^{\prime}$ & $\mathrm{x}_{i}^{\prime}$ & $\mathrm{y}_{i}^{\prime}$ & $\mathrm{z}_{i}$ & $\mathbf{s}_{i j}^{\prime}$ & $\mathrm{x}_{i}$ & $\mathrm{y}_{i}$ & $\mathrm{z}_{i}$ \\
\hline $\mathbf{s}_{41}^{\prime}$ & 0 & 0 & 0 & $\mathbf{s}_{23}^{\prime}$ & 0 & -12.2 & 0 \\
$\mathbf{s}_{43}^{\prime}$ & -4 & -8.5 & 0 & $\mathbf{s}_{32}^{\prime}$ & 0 & -7.4 & 0 \\
$\mathbf{s}_{12}^{\prime}$ & 2 & 0 & 0 & & & & \\
\hline
\end{tabular}

Estimates of generalized coordinates are $\mathbf{q}=\left[\begin{array}{lllll}0^{\circ} & 180^{\circ} & 227.6^{\circ} & 208.3^{\circ}\end{array}\right]^{T}$. For a given initial estimate that does not satisfy the constraint function $\Phi$, the Moore-Penrose iteration method is used to obtain an initial assembly. The iterative algorithm is continued until the magnitudes of all errors and changes in approximate solutions satisfy $\left\|\Phi_{k}^{s p h}\left(\mathbf{q}^{(i)}\right)\right\| \leq \varepsilon_{e}$ and $\left|q_{j}^{(i)}-q_{j}^{(i-1)}\right| \leq \varepsilon_{s}$, where $\varepsilon_{e}$ and $\varepsilon_{s}$ are two small numbers, $k$ is the number of constraint equations, $j$ is the number of generalized coordinates, and $i$ is the iteration number. The Jacobian matrix $\Phi_{\mathbf{q}}^{s p h}$ in joint coordinate space transformed from the Cartesian space is

$$
\Phi_{\mathbf{q}}^{s p h}=\left[\begin{array}{lll}
\Phi_{\hat{\mathbf{z}}_{2}}^{s p h} \mathbf{B}_{1} & \Phi_{\hat{\mathbf{z}}_{2}}^{s p h} \mathbf{B}_{2} & \Phi_{\hat{\mathbf{z}}_{3}}^{s p h} \mathbf{B}_{3}
\end{array}\right]
$$

The Jacobian matrix in the Cartesian space for the spherical joint, is given as

and

$$
\begin{gathered}
\Phi_{\hat{\mathbf{z}}_{2}}^{s p h}=\left[\begin{array}{ll}
-\mathbf{I} & \widetilde{\mathbf{r}}_{2}+\widetilde{\mathbf{S}}_{23}
\end{array}\right] \\
\Phi_{\hat{\mathbf{z}}_{2}}^{s p h}=-\Phi_{\hat{\mathbf{z}}_{3}}^{s p h}
\end{gathered}
$$

where $\mathbf{B}_{1}, \mathbf{B}_{2}$, and $\mathbf{B}_{3}$ are velocity transformation matrices for the revolute joint, universal joint, and revolute joint, respectively, such that

$$
\mathbf{B}_{1}=\left[\begin{array}{c}
\tilde{\mathbf{r}}_{1} \mathbf{h}_{41} \\
\mathbf{h}_{41}
\end{array}\right] \quad \mathbf{B}_{2}=\left[\begin{array}{cc}
\tilde{\mathbf{r}}_{\mathbf{h}} \mathbf{h}_{12} & \tilde{\mathbf{r}}_{2} \mathbf{g}_{12} \\
\mathbf{h}_{12} & \mathbf{g}_{12}
\end{array}\right] \quad \mathbf{B}_{3}=\left[\begin{array}{c}
\tilde{\mathbf{r}}_{3} \mathbf{h}_{43} \\
\mathbf{h}_{43}
\end{array}\right]
$$

where $\mathbf{h}_{41}$ and $\mathbf{h}_{43}$ are unit vectors along the rotational joint axes in the global reference frame which can be expressed by

and

$$
\begin{gathered}
\mathbf{h}_{41}=\mathbf{A}_{4} \mathbf{C}_{41}\left[\begin{array}{lll}
0 & 0 & 1
\end{array}\right]^{T} \\
\mathbf{C}_{41}=\left[\begin{array}{ccc}
0 & 0 & 1 \\
0 & -1 & 0 \\
1 & 0 & 0
\end{array}\right] \\
\mathbf{h}_{43}=\mathbf{A}_{4} \mathbf{C}_{43}\left[\begin{array}{lll}
0 & 0 & 1
\end{array}\right]^{T}
\end{gathered}
$$




$$
\mathbf{C}_{43}=\left[\begin{array}{ccc}
1 & 0 & 0 \\
0 & 0 & -1 \\
0 & 1 & 0
\end{array}\right]
$$

where $\mathbf{h}_{12}$ and $\mathbf{g}_{12}$ are two unit vectors along the rotational axes $\mathbf{z}_{12}^{\prime \prime}$ and $\mathbf{y}_{12}^{\prime \prime}$ in the global reference frame defined as

$$
\begin{gathered}
\mathbf{h}_{12}=\mathbf{A}_{1} \mathbf{C}_{12}\left[\begin{array}{lll}
0 & 0 & 1
\end{array}\right]^{T} \\
\mathbf{C}_{12}=\left[\begin{array}{ccc}
0.9293 & 0.3693 & 0 \\
-0.2768 & 0.6967 & 0.6618 \\
0.2444 & -0.6150 & 0.7497
\end{array}\right] \\
\mathbf{g}_{12}=\mathbf{A}_{1} \mathbf{C}_{12} \mathbf{A}_{121}^{\prime \prime}\left(q_{2}\right)\left[\begin{array}{lll}
0 & 1 & 0
\end{array}\right]^{\mathrm{T}}
\end{gathered}
$$

and the rotation matrix $\mathbf{A}_{121}^{\prime \prime}$ is written as

$$
\mathbf{A}_{121}^{\prime \prime}\left(q_{2}\right)=\left[\begin{array}{ccc}
\cos q_{2} & -\sin q_{2} & 0 \\
\sin q_{2} & \cos q_{2} & 0 \\
0 & 0 & 1
\end{array}\right]
$$

and the variational generalized coordinates are $\delta \mathbf{q}=\left[\begin{array}{llll}\delta q_{1} & \delta q_{2} & \delta q_{3} & \delta q_{4}\end{array}\right]^{T}$. Using the MoorePenrose method to update the generalized coordinates $\mathbf{q}$, only one iteration is required and the set of new generalized coordinates are $\mathbf{q}=\left[\begin{array}{llll}0.0764^{\circ} & 179.8572^{\circ} & 227.6018^{\circ} & 208.1882^{\circ}\end{array}\right]^{T}$.

After initial assembly, the joint-attachment vectors $\mathbf{s}_{i j}^{\prime}$ and rotation vector $\xi_{i j}$ (or the Euler parameter vector $\mathbf{p}$ ) are chosen as design parameters.

In the spatial four-bar mechanism, the design parameters of joint-attachment vectors in body-fixed reference frame are $\mathbf{s}_{41}^{\prime}, \mathbf{s}_{43}^{\prime}, \mathbf{s}_{12}^{\prime}, \mathbf{s}_{23}^{\prime}$ and $\mathbf{s}_{32}^{\prime}$. Once the configuration changes, the constraint equations are violated, and there are three methods to be performed until constraint violations are satisfied to assemble the mechanism.

(1) The generalized coordinates $\mathbf{q}$ are changed such that

$$
\Phi_{\hat{\mathbf{z}}_{2}}^{s p h} \mathbf{B}_{1} \delta q_{1}+\Phi_{\hat{\mathbf{z}}_{2}}^{s p h} \mathbf{B}_{2}\left[\begin{array}{l}
\delta q_{2} \\
\delta q_{3}
\end{array}\right]+\Phi_{\hat{\mathbf{z}}_{3}}^{s p h} \mathbf{B}_{3} \delta q_{4}=-\Phi^{s p h}
$$

A designer, however, may choose to maintain the generalized coordinates as constants but elect to allow for a change in the dimension of each body which gives rise to the following methods.

(2) The joint attachment vectors $\mathbf{s}_{i j}^{\prime}$ are changed such that

$$
\Phi_{\hat{\mathbf{z}}_{2}}^{s p h} \mathbf{M}_{4} \delta \mathbf{s}_{41}^{\prime}+\Phi_{\hat{\mathbf{z}}_{2}}^{s p h} \mathbf{M}_{1} \delta \mathbf{s}_{12}^{\prime}+\Phi_{\hat{\mathbf{z}}_{3}}^{s p h} \mathbf{M}_{4} \delta \mathbf{s}_{43}^{\prime}+\Phi_{\mathbf{s}_{23}^{\prime}}^{s p h} \delta \mathbf{s}_{23}^{\prime}+\Phi_{\mathbf{s}_{32}^{\prime}}^{s p h} \delta \mathbf{s}_{32}^{\prime}=-\Phi^{s p h}
$$

where

$$
\begin{aligned}
& \mathbf{M}_{1}=\left[\begin{array}{c}
\mathbf{A}_{1} \\
\mathbf{0}
\end{array}\right] \quad \mathbf{M}_{4}=\left[\begin{array}{c}
\mathbf{A}_{4} \\
\mathbf{0}
\end{array}\right] \\
& \Phi_{\mathbf{s}_{23}^{\prime}}^{s p h}=-\mathbf{A}_{2}
\end{aligned}
$$




$$
\Phi_{\mathbf{s}_{32}}^{s p h}=\mathbf{A}_{3}
$$

3) The generalized coordinates $\mathbf{q}$ and joint-attachment vectors $\mathbf{s}_{i j}^{\prime}$ are changed such that

$$
\begin{aligned}
\Phi_{\hat{\mathbf{z}}_{2}}^{s p h} \mathbf{B}_{1} \delta q_{1}+\Phi_{\hat{\mathbf{z}}_{2}}^{s p h} \mathbf{B}_{2}\left[\begin{array}{c}
\delta q_{2} \\
\delta q_{3}
\end{array}\right] & +\Phi_{\hat{\mathbf{z}}_{2}}^{s p h} \mathbf{M}_{4} \delta \mathbf{s}_{41}^{\prime}+\Phi_{\hat{\mathbf{z}}_{2}}^{s p h} \mathbf{M}_{1} \delta \mathbf{s}_{12}^{\prime}+\Phi_{\hat{\mathbf{z}}_{3}}^{s p h} \mathbf{B}_{3} \delta q_{4}+\Phi_{\hat{\mathbf{z}}_{3}}^{s p h} \mathbf{M}_{4} \delta \mathbf{s}_{43}^{\prime} \\
& +\Phi_{\mathbf{s}_{23}}^{s p h} \delta_{\mathbf{s}_{23}}^{\prime}+\Phi_{\mathbf{s}_{32}}^{s p h} \delta \mathbf{s}_{32}^{\prime}=-\Phi^{s p h}
\end{aligned}
$$

Simulation results due to changing $\mathbf{s}_{23}^{\prime}$ from $\left[\begin{array}{lll}0 & -12.2 & 0\end{array}\right]^{T}$ to $\left[\begin{array}{lll}0 & -10 & 0\end{array}\right]^{T}$ are presented in Table 2. Column 2 presents the initial estimates for a general configuration. An initial assembly is computed and entered into column 3. Using Eq. (57), only the generalized coordinates are allowed to change and those values computed for an assembled configuration are shown in column 4. If only the joint-attachment vectors are allowed to change using Eq. (58), the computed assembly is entered into column 5 of Table 2. Figure 4a depicts a schematic of the mechanism after computing the new joint coordinates (method 1). Note that only joint angles have changed. Figure $4 \mathrm{~b}$ depicts a schematic of the mechanism after computing only new jointattachment vectors. Note that, even for a very small tolerance (a norm of $\sim 10^{-6}$ ), the method exhibits a high rate of convergence (within 4 iterations).

\begin{tabular}{|c|c|c|c|c|c|}
\hline $\begin{array}{c}1 \\
\text { variables }\end{array}$ & $\begin{array}{c}2 \\
\text { initial estimates }\end{array}$ & $\begin{array}{c}3 \\
\text { initial assembly }\end{array}$ & $\begin{array}{l}4 \\
\mathbf{q}\end{array}$ & $\begin{array}{c}5 \\
\mathbf{s}_{i j}^{\prime}\end{array}$ & $\begin{array}{c}6 \\
\mathbf{q}, \mathbf{s}_{i j}^{\prime}\end{array}$ \\
\hline $\mathrm{q}_{1}$ & 0 & 0.0764 & 28.9745 & & 15.3243 \\
\hline$q_{2}$ & 180 & 179.8572 & 142.6831 & & 160.2042 \\
\hline $\mathrm{q}_{3}$ & 227.6 & 227.6018 & 227.4333 & & 227.5725 \\
\hline $\mathrm{q}_{4}$ & 208.3 & 208.1882 & 175.4225 & & 190.5807 \\
\hline $\mathbf{s}_{41}^{\prime}$ & {$\left[\begin{array}{l}0 \\
0 \\
0\end{array}\right]$} & & & {$\left[\begin{array}{c}-0.3379 \\
-0.3831 \\
0.2039\end{array}\right]$} & {$\left[\begin{array}{c}-0.1102 \\
-0.1948 \\
0.1181\end{array}\right]$} \\
\hline $\mathbf{s}_{43}$ & {$\left[\begin{array}{c}-4 \\
-8.5 \\
0\end{array}\right]$} & & & {$\left[\begin{array}{l}-3.6621 \\
-8.1169 \\
-0.2039\end{array}\right]$} & {$\left[\begin{array}{l}-3.8898 \\
-8.3052 \\
-0.1181\end{array}\right]$} \\
\hline $\mathbf{s}_{12}$ & {$\left[\begin{array}{l}2 \\
0 \\
0\end{array}\right]$} & & & {$\left[\begin{array}{c}2.2044 \\
0.3828 \\
-0.3379\end{array}\right]$} & {$\left[\begin{array}{c}2.1175 \\
0.1947 \\
-0.1102\end{array}\right]$} \\
\hline $\overrightarrow{\mathbf{s}_{23}^{\prime}}$ & {$\left[\begin{array}{llll}0 & -12.2 & 0\end{array}\right]^{\mathrm{T}}$} & & {$\left[\begin{array}{lll}0 & -10 & 0\end{array}\right]^{T}$} & {$\left[\begin{array}{lll}0 & -10 & 0\end{array}\right]^{T}$} & {$\left[\begin{array}{lll}0 & -10 & 0\end{array}\right]^{T}$} \\
\hline $\mathbf{s}_{32}$ & $\left.\begin{array}{c}0 \\
-7.4 \\
0\end{array}\right]$ & & & {$\left[\begin{array}{l}-0.2015 \\
-7.0607 \\
-0.3831\end{array}\right]$} & {$\left[\begin{array}{l}-0.0416 \\
-7.2443 \\
-0.1948\end{array}\right]$} \\
\hline $\mathbf{P}_{1}$ & {$\left[\begin{array}{c}0 \\
0.7071 \\
0.7071 \\
0\end{array}\right]$} & & & & \\
\hline $\mathbf{P}_{3}$ & {$\left[\begin{array}{c}0.7071 \\
0.7071 \\
0 \\
0\end{array}\right]$} & & & & \\
\hline
\end{tabular}

TABLE 2

Simulation results due to changing $\mathbf{S}_{23}$ 


\begin{tabular}{|c|c|c|c|c|c|}
\hline $\mathbf{P}_{2}$ & {$\left[\begin{array}{c}0.9187 \\
-0.3475 \\
-0.0665 \\
-0.1758\end{array}\right]$} & & & & \\
\hline$\Phi_{\text {norm }}^{\mathrm{s}}$ & & $1.2547 \mathrm{e}-05$ & $7.3779 \mathrm{e}-05$ & $2.1756 \mathrm{e}-15$ & $6.1485 \mathrm{e}-07$ \\
\hline Iteration number & & 1 & 4 & 2 & 4 \\
\hline
\end{tabular}
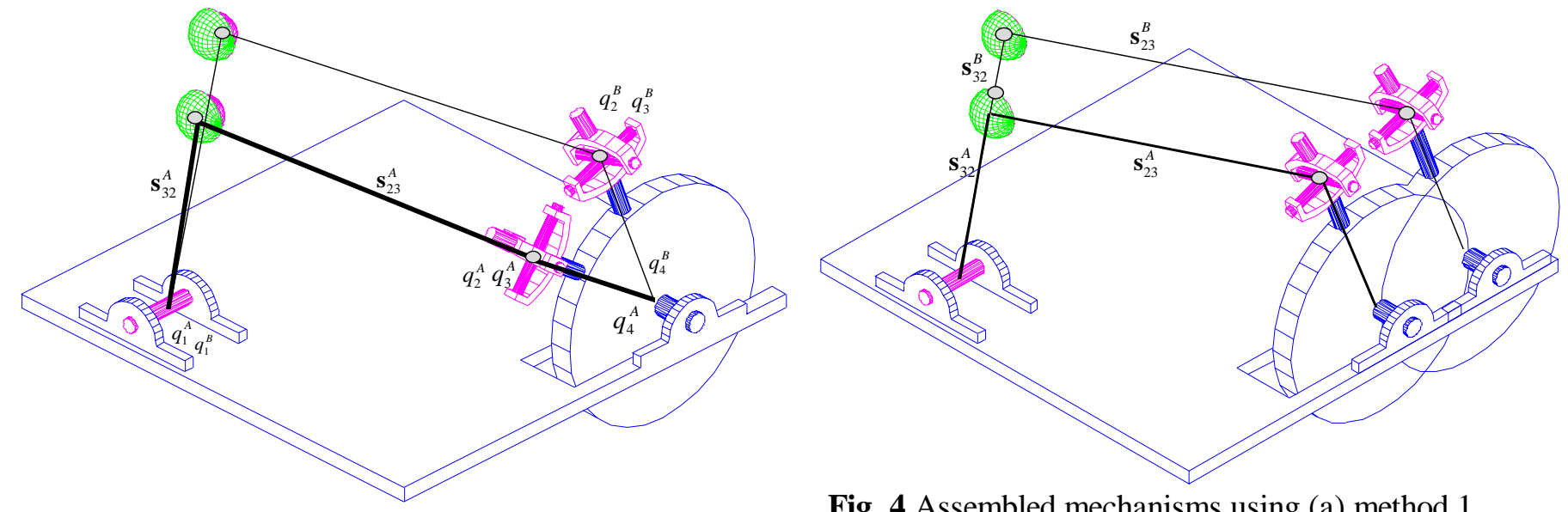

Fig. 4 Assembled mechanisms using (a) method 1 and (b) method 2

If the length of link 2 , however, is reduced from 12.2 to 6 , it is geometrically impossible that an assembled configuration be obtained since link 2 and link 3 cannot be connected by a spherical joint. Nevertheless, an assembled configuration can be obtained using the other two methods. In fact, it has been our experience that methods (2) and (3) are more practical from a design point of view.

Another possibility arises for which the Euler parameters can be changed. In such a case, the orientation of a body can be altered, i.e., varying $\mathbf{p}_{1}, \mathbf{p}_{2}$ and $\mathbf{p}_{3}$. There are three practical methods to be performed.

1) The generalized coordinates $\mathbf{q}$ are changed (Eq. 57).

2) The generalized coordinates $\mathbf{q}$ and joint attachment vectors $\mathbf{s}_{i j}^{\prime}$ are changed (Eq. 58).

3) The generalized coordinates $\mathbf{q}$, joint attachment vectors $\mathbf{s}_{i j}^{\prime}$, and Euler parameters $\mathbf{p}_{j}$ are changed such that

$$
\begin{gathered}
\Phi_{\hat{\mathbf{z}}_{2}}^{s p h} \mathbf{B}_{1} \delta q_{1}+\Phi_{\hat{\mathbf{z}}_{2}}^{s p h} \mathbf{B}_{2}\left[\begin{array}{c}
\delta q_{2} \\
\delta q_{3}
\end{array}\right]+\Phi_{\hat{\mathbf{z}}_{2}}^{s p h} \mathbf{M}_{4} \delta \mathbf{s}_{41}^{\prime}+\Phi_{\hat{\mathbf{z}}_{2}}^{s p h} \mathbf{M}_{1} \delta \mathbf{s}_{12}^{\prime}+\Phi_{\hat{\mathbf{z}}_{2}}^{s p h} \mathbf{N}_{1}\left(2 \mathbf{A}_{4} \mathbf{E}_{1}\right) \delta \mathbf{p}_{1}+\Phi_{\hat{\mathbf{z}}_{2}}^{s p h} \mathbf{N}_{2}\left(2 \mathbf{A}_{1} \mathbf{E}_{2}\right) \delta \mathbf{p}_{2} \\
\Phi_{\hat{\mathbf{z}}_{3}}^{s p h} \mathbf{B}_{3} \delta q_{4}+\Phi_{\hat{\mathbf{z}}_{3}}^{s p h} \mathbf{M}_{4} \delta \mathbf{s}_{43}^{\prime}+\Phi_{\hat{\mathbf{z}}_{3}}^{s p h} \mathbf{N}_{3}\left(2 \mathbf{A}_{4} \mathbf{E}_{3}\right) \delta \mathbf{p}_{3}+\Phi_{\mathbf{s}_{23}}^{s p h} \delta \mathbf{s}_{23}^{\prime}+\Phi_{\mathbf{s}_{32}}^{s p h} \mathbf{s}_{32}^{\prime}=-\Phi^{s p h}
\end{gathered}
$$

where

$$
\mathbf{N}_{1}=\left[\begin{array}{c}
\widetilde{\mathbf{r}}_{1} \\
\mathbf{I}
\end{array}\right], \mathbf{N}_{2}=\left[\begin{array}{c}
\widetilde{\mathbf{r}}_{2} \\
\mathbf{I}
\end{array}\right], \mathbf{N}_{3}=\left[\begin{array}{c}
\widetilde{\mathbf{r}}_{3} \\
\mathbf{I}
\end{array}\right]
$$


Euler parameters $\mathbf{p}_{3}$ are changed from $\left[\begin{array}{llllll}0.7071 & 0.7071 & 0 & 0\end{array}\right]^{\mathrm{T}}$ to $\left[\begin{array}{llll}0 & 0.7071 & 0.7071 & 0\end{array}\right]^{\mathrm{T}}$, thus the corresponding transformation matrix $\mathbf{C}_{43}$ from revolute joint reference frame to ground reference frame is changed from

$$
\left[\begin{array}{ccc}
1 & 0 & 0 \\
0 & 0 & -1 \\
0 & 1 & 0
\end{array}\right] \text { to }\left[\begin{array}{ccc}
0 & 0 & 1 \\
0 & -1 & 0 \\
1 & 0 & 0
\end{array}\right]
$$

Using the three methods described above, the system can be assembled satisfactorily. The results are presented in Table 3. Initial estimates are entered into column 2. An initial assembly is computed and entered into column 3. For a change in the link orientations while keeping constant joint-attachment vectors, the generalized coordinates are computed and entered in column 4. Note that 34 iterations were required to compute q. If the generalized coordinates and the jointattachment vectors are computed using Eq. (58), only 12 iterations are needed to converge to an assembled configuration the results of which are entered into column 5. If the generalized variables, the joint-attachment vectors, and the Euler parameters are computed using Eq. (63), only 8 iterations are needed to converge to a solution. The results are entered into column 6 .

\section{TABLE 3}

Simulation results due to changing $\mathbf{p}_{3}$

\begin{tabular}{|c|c|c|c|c|c|}
\hline $\begin{array}{c}1 \\
\text { variables }\end{array}$ & $\begin{array}{c}2 \\
\text { initial estimates }\end{array}$ & $\begin{array}{c}3 \\
\text { initial assembly }\end{array}$ & $\begin{array}{l}4 \\
\mathbf{q}\end{array}$ & $\begin{array}{c}5 \\
\mathbf{q}, \mathbf{s}_{i j}^{\prime}\end{array}$ & $\begin{array}{c}6 \\
\mathbf{q}, \mathbf{s}_{i j}^{\prime} \text { and } \mathbf{p}_{j}\end{array}$ \\
\hline$q_{1}$ & 0 & 0.0764 & 247.9598 & -64.4176 & 4.7796 \\
\hline$q_{2}$ & 180 & 179.8572 & -44.4288 & 281.3515 & 190.5383 \\
\hline$q_{3}$ & 227.6 & 227.6018 & -224.4808 & 55.9736 & 224.9965 \\
\hline$q_{4}$ & 208.3 & 208.1882 & 190.7124 & 120.2919 & 200.8773 \\
\hline $\mathbf{S}_{41}^{\prime}$ & {$\left[\begin{array}{l}0 \\
0 \\
0\end{array}\right]$} & & & {$\left[\begin{array}{c}-0.0670 \\
0.3966 \\
0.4846\end{array}\right]$} & {$\left[\begin{array}{l}-0.1429 \\
-0.2572 \\
-0.0608\end{array}\right]$} \\
\hline $\mathbf{S}_{43}^{\prime}$ & {$\left[\begin{array}{c}-4 \\
-8.5 \\
0\end{array}\right]$} & & & {$\left[\begin{array}{l}-3.9331 \\
-8.8966 \\
-0.4846\end{array}\right]$} & $\left.\begin{array}{c}-3.8571 \\
-8.2428 \\
0.0608\end{array}\right]$ \\
\hline $\mathbf{s}_{12}^{\prime}$ & {$\left[\begin{array}{l}2 \\
0 \\
0\end{array}\right]$} & & & {$\left[\begin{array}{c}2.9719 \\
-0.2223 \\
-0.0670\end{array}\right]$} & $\left.\begin{array}{c}1.7320 \\
-0.0752 \\
-0.0214\end{array}\right]$ \\
\hline $\mathbf{S}_{23}^{\prime}$ & {$\left[\begin{array}{llll}0 & -12.2 & 0\end{array}\right]^{\mathrm{T}}$} & & & {$\left[\begin{array}{c}0.3487 \\
-7.2995 \\
0.0077\end{array}\right]$} & $\left.\begin{array}{l}-0.2337 \\
-11.976 \\
-0.0508\end{array}\right]$ \\
\hline$\overline{\mathbf{s}_{32}^{\prime}}$ & $\left.\begin{array}{c}0 \\
-7.4 \\
0\end{array}\right]$ & & & {$\left[\begin{array}{c}-0.2023 \\
-5.9993 \\
0.4846\end{array}\right]$} & $\left.\begin{array}{l}-0.0153 \\
-7.3014 \\
-0.0608\end{array}\right]$ \\
\hline $\mathbf{P}_{1}$ & {$\left[\begin{array}{c}0 \\
0.7071 \\
0.7071 \\
0\end{array}\right]$} & & & & {$\left[\begin{array}{c}-0.1111 \\
0.4933 \\
05690 \\
0.6485\end{array}\right]$} \\
\hline $\mathbf{P}_{3}$ & {$\left[\begin{array}{c}0.7071 \\
0.7071 \\
0 \\
0\end{array}\right]$} & & {$\left[\begin{array}{c}0 \\
0.7071 \\
0.7071 \\
0\end{array}\right]$} & {$\left[\begin{array}{c}0 \\
0.7071 \\
0.7071 \\
0\end{array}\right]$} & {$\left[\begin{array}{c}0 \\
0.7071 \\
0.7071 \\
0\end{array}\right]$} \\
\hline
\end{tabular}




\begin{tabular}{|c|c|c|c|c|c|}
\hline $\mathbf{P}_{2}$ & {$\left[\begin{array}{c}0.9187 \\
-0.3475 \\
-0.0665 \\
-0.1758\end{array}\right]$} & & & & {$\left[\begin{array}{c}0.8759 \\
-0.4289 \\
0.1490 \\
0.1631\end{array}\right]$} \\
\hline$\Phi_{\text {norm }}^{\mathrm{s}}$ & & $1.2547 \mathrm{e}-05$ & $1.1235 \mathrm{e}-4$ & $4.8947 \mathrm{e}-07$ & $2.7985 \mathrm{e}-06$ \\
\hline Iteration number & & 1 & 34 & 12 & 8 \\
\hline
\end{tabular}

It can be noticed, in this case as in most cases that have been implemented using this experimental code, that allowing the code to compute generalized coordinates, joint attachmentvectors, and Euler parameters (method 3), exhibits the highest rate of convergence.

\section{CONCLUSIONS}

An analytical formulation for studying the kinematic sensitivity of the spatial four-bar mechanism is presented. The formulation presented in this paper and illustrated by a numerical example demonstrates the validity of a general purpose formulation and experimental computer code for the computation of design parameters after an alteration in the original design.

The spatial four-bar mechanism is modeled using graph theory and closed loops are converted to a tree structure using a cut-joint constraint formulation. It was shown that this formulation can be derived with respect to design parameters to obtain kinematic sensitivity. These constraints are derived keeping the joint-attachment vectors and orientation matrices (Euler parameters) as design variables. Variations of these vectors and matrices were also developed.

It was shown that the Jacobian matrix in Cartesian space can be transformed to joint coordinate space to compute variations. It was shown that starting from an initial configuration that satisfies the constraints, applying a design change to the joint-attachment vectors or Euler parameters, can be propagated throughout the mechanism. It was shown that an assembled configuration can be obtained using the Moore-Penrose pseudo inverse method. The method has showed to be applicable for a variety of design parameters. It has been shown that the method of changing linkage lengths while maintaining constant generalized coordinates is the fastest method to obtain a new set of variables. This method has also exhibited the highest accuracy.

The kinematic sensitivity due to changing the orientation of a body has showed a higher rate of convergence to an assembled configuration using the method of allowing the generalized coordinates, joint-attachment vectors, and Euler parameters to be automatically computed. It has been our experience that allowing the maximum number of design parameters to change, results in higher potential for convergence.

\section{ACKNOWLEDGMENTS}

This research was funded by the US Army Tank Automotive Command Center (TACOM) through the Automotive Research Center (Department of Defense contract number DAAE07-94- 
C-R094). The authors also acknowledge Professor E. J. Haug's insightful comments and the reviewers for their valuable comments.

\section{REFERENCES}

1. R. M. Bodduluri and J. M. McCarthy, Journal of Mechanical Design, Transactions of the ASME 114: 55-60 (1992).

2. C. W. Wampler, A. P. Morgan, and A. J. Sommese, Journal of Mechanical Design, Transactions of the ASME, 114: 153-159 (1992).

3. T. Subbian and D. R. Flugrad, Journal of Mechanical Design, Transactions of the ASME, 115: 262-268 (1993).

4. T. W. Norton, A. Midha, and L. L. Howell, Journal of Mechanical Design, Transactions of the ASME, 116: 1132-1140 (1994).

5. S. S. Khanuja, G. A. Mettlash, and A. Midha, Proceedings of the 1994 ASME Design Technical Conference, Minneapolis, MN, pp. 229-237.

6. W. M. Hwang and C. F. Chang, Transactions of the Canadian Society for Mechanical Engineering, 18: 19-33 (1994).

7. V. Uhruh and P. Krishnaswami, Journal of Mechanical Design, Transactions of the ASME, 117: 143-149 (1995).

8. R. Yang and P. S. Krishnaprasad, Dynamics and Stability of Systems, 9: 19-45 (1994).

9. K. Kazerounian and R. Solecki, Mechanism and Machine Theory, 28: 437-445 (1993).

10. E. Soylemez, Mechanisms and Machine Theory, 28: 539-552 (1993).

11. Z. Liu and J. Angeles, Journal of Mechanical Design, Transactions of the ASME, 114: 574579 (1992).

12. Z. Liu and J. Angeles Journal of Mechanical Design, Transactions of the ASME, 114: 569573 (1992).

13. J. Angeles and Z. Liu, Journal of Mechanical Design, Transactions of the ASME, 114: 394405 (1992).

14. P. N. Sheth and J. J. Uicker, Journal of Engineering for Industry, 94: 454-464 (1972).

15. J. Wittenburg, Dynamics of Systems of Rigid Bodies, (B G Teubner), Stuttgart, 1977.

16. J. Wittenburg and U. Wolz, MESA VERDE: a symbolic program for nonlinear articulatedrigid-body, ASME, 85-DET-151.

17. D. S. Bae and E. J. Haug, Mechanics of Structures and Machines, 15 (1987).

18. D. S. Bae and E. J. Haug, Mechanics of Structures and Machines, 15 (1987).

19. R. S. Hwang, D. S. Bae, J. G. Kuhl, and E. J. Haug, Journal of Mechanisms, Transmissions, and Automation in Design, (1988).

20. R. S. Hwang, and E. J. Haug, Technical Report R-13, Center for Simulation and Design Optimization, the University of Iowa, Iowa City, IA (1988).

21. E. J. Haug, Computer Aided Kinematics and Dynamics of Mechanical Systems Vol I Allyn \& Bacon (1989).

22. F.F. Tsai and E.J. Haug Automated Technical Methods for High Speed Simulation of Multibody Dynamic Systems, Technical Report R-47, Center for Computer-Aided Design, The University of Iowa, Iowa City, IA (1989). 
23. H. L. Zou, K. Abdel-Malek, and J. Y. Wang, ASME Journal of Mechanical Design (submitted). 